Eur. J. Clin. Chem. Clin. Biochem.

Vol. 30, 1992, pp. $779-784$

(C) 1992 Walter de Gruyter \& Co. Berlin - New York

\title{
Creatinine and Automatic Analysers in Relation to Icteric Specimens
}

\author{
By B. G. Blijenberg, E. C. Liesting and L. Zwang
}

Department of Clinical Chemistry, Academic Hospital Rotterdam-Dijkzigt, Rotterdam, The Netherlands

(Received December 12, 1991/June 9, 1992)

Summary: A study is described on the interference of bilirubin with the determination of creatinine in serum using three automatic analysers i. e. the Technicon Chem-1, the DuPont Dimension and the Baxter Paramax. In all instruments a kinetic Jaffé reaction is applied. As comparison method we used a HPLC based selected method.

All methods proved to be sensitive to interference, the Paramax showing the best performance with our set of specimens.

Results regarding modifications leading to improvement and possible future experiments are discussed.

\section{Introduction}

Soon after the introduction of the Technicon Chem-1 clinical chemical analyser in our laboratory, we became involved in discussions with clinicians on the accuracy of our new creatinine determination, which was based on the Jaffé reaction and modified as a continuous procedure. On closer investigation, it was found that all questionable results could be traced to icteric specimens. The interference of bilirubin with the creatinine determination is very well known and was, of course, also known to us, not only in relation to our evaluation study (1). However, our investigations were restricted to bilirubin-enriched specimens, and therefore to the effects of unconjugated bilirubin, whereas in daily practice elevated bilirubin is usually due to conjugated bilirubin. Furthermore, a number of drugs are also known to interfere. Therefore we performed a small pilot study with very icteric specimens (total bilirubin varying from 100 to $500 \mu \mathrm{mol} / \mathrm{l}$ ) in order to obtain more background information on creatinine and automatic analysers in relation to icteric specimens. As a reference method, we were only able at that time to use a Fuller's earth modification. The results were indeed disturbing as can be seen in figure 1.
We made contact with the Technicon company where this problem had been under study for some time. A new reagent formulation was about to be released. We started a thorough study of our own in the hope of deciding which method should be used for creatinine for both non-icteric and icteric sera on a $24 \mathrm{~h}$ per day basis. Did the problem occur in all icteric specimens, and was it possible to correct it? Since a DuPont Dimension was also in operation in our laboratory, we considered whether this instrument might produce better results.

In the meantime we were invited by Technicon to evaluate their modification. In view of reported inconstancies in the Fuller's earth reagent (2), we first developed a sound reference method for creatinine. This method, based on HPLC, has been described elsewhere (3).

We also included the Baxter Paramax analyser, in order to study a more elaborate version of the state of the art creatinine methodology. It appears that the principle used by all manufacturers is the measurement of the rate of the Jaffe reaction.

Later, we were asked by DuPont (The Netherlands) to compare an open channel Dimension version with other instruments using our serum bank, because of critical remarks they had received from customers. 


\section{Materials and Methods}

\section{Materials}

Creatinine, bilirubin, ascorbic acid and acetone were obtained from Merck (Germany), human albumin from Behring Werke (Germany).

\section{Methods}

As reference method we used a HPLC-based method. Details will be described elsewhere (3).

Instrumentation: all analysers, Paramax (Baxter, U.S.A.), Dimension (DuPont, U.S.A.) and Chem-1 (Bayer-Technicon, Germany) were operated according to the manufacturers' instructions. The Paramax was calibrated with the Baxter Comprehensive Calibrators, levels I and II. The Dimension was calibrated with patient samples using the Chem-1 results. The Chem-1 was calibrated with the Setpoint Chem-1 Calibrator modified as described (4).

A preliminary report on the new Chem-1 creatinine method was presented at the combined IFCC-AACC meeting in 1990 in San Francisco (5). Briefly, the reagent system was modified (reversal of picrate and base solutions), together with the inclusion of a selective bichromatic correction. The other two analysers use a parallel bichromatic algorithm.

The modified Dimension procedure using an open channel is a two-step method, whereas the commercial version is a one-step method. It is comparable to the method Du Pont used about two years ago, but abandoned because it was too slow.

All interference experiments were performed according to the CERMAB procedure, except that an albumin solution was used instead of a serum pool (6).

\section{Patient samples}

All serum specimens were from various departments in our hospital. Selection for study was based only on colour. All sera were characterized by measuring the total bilirubin content with the Chem-1. A distribution of these bilirubin levels of the samples in the various comparisons is given in table 1 . The sera were stored at $-80^{\circ} \mathrm{C}$ until analysis.

Tab. 1. Frequency distribution in relation to the total bilirubin concentrations

\begin{tabular}{lrrr}
\hline $\begin{array}{l}\text { Concentration range } \\
(\mu \mathrm{mol} / \mathrm{l})\end{array}$ & \multicolumn{3}{c}{ Number of specimens } \\
\cline { 2 - 4 } & $\mathrm{A}$ & $\mathrm{B}$ & $\mathrm{C}$ \\
\hline$<100$ & 3 & 25 & 3 \\
$101-150$ & 32 & 37 & 33 \\
$151-200$ & 22 & 30 & 23 \\
$201-250$ & 8 & 10 & 9 \\
$251-300$ & 6 & 13 & 7 \\
$301-350$ & 4 & 8 & 4 \\
$351-400$ & 4 & 13 & 7 \\
$>400$ & 6 & 7 & 13 \\
Total & 85 & 144 & 99 \\
\hline
\end{tabular}

$\mathrm{A}=$ comparison of Paramax, Dimension and Chem-1 (current formulation), see Materials and Methods

$\mathrm{B}=$ comparison of current and new Chem-1 formulation, see Materials and Methods

$\mathrm{C}=$ comparison of both Dimension versions, see Materials and Methods

\section{Results}

Comparison Paramax, Dimension and Chem-1

For the comparison of the three analysers we used 85 icteric specimens. The total bilirubin content ranged from 89 to $532 \mu \mathrm{mol} / 1$ with a distribution as given in table 1. In figures 2, 3 and 4 the results are compared with those obtained by HPLC.

In addition to these patient samples, we studied the influence of unconjugated bilirubin by enriching a human albumin solution (6). The results of these measurements are depicted in figure 5.

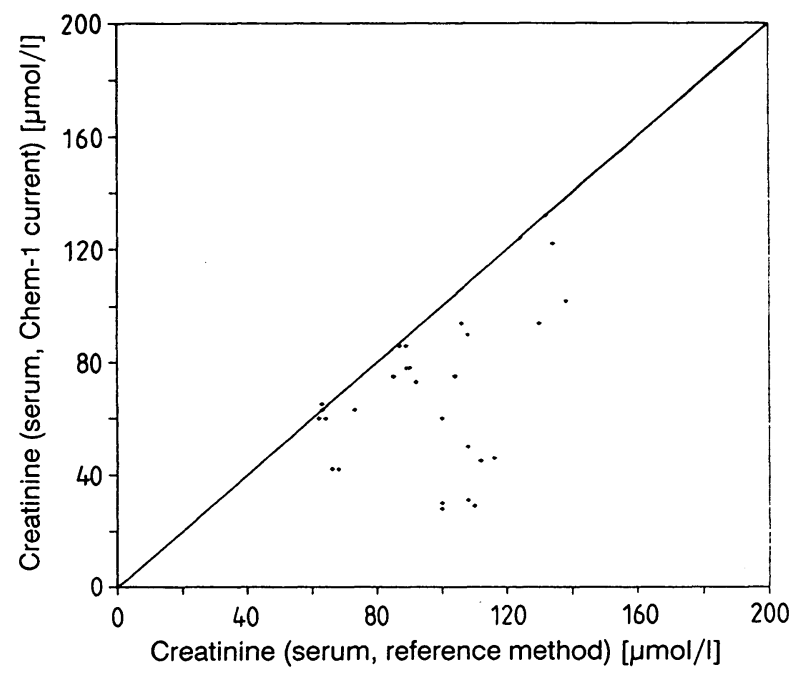

Fig. 1. Comparison of the current Chem-1 creatinine formulation (y-axis) and a Fuller's earth based manual reference method (x-axis) with 30 icteric samples (total bilirubin ranging from about 100 till about $500 \mu \mathrm{mol} / \mathrm{l})$.

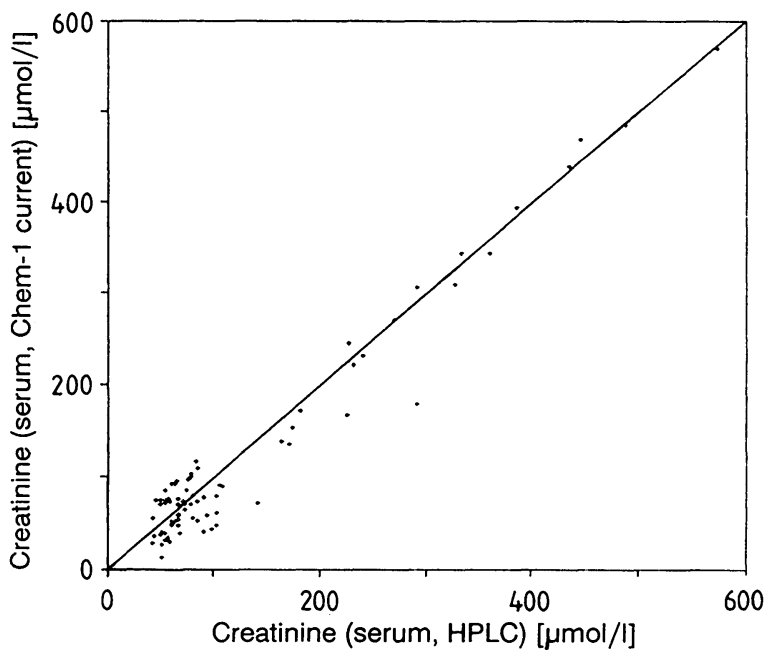

Fig. 2. Comparison of the current Chem-1 creatinine formulation ( $y$-axis) and a HPLC based reference method ( $x$ axis) with 85 icteric samples (see Results). 


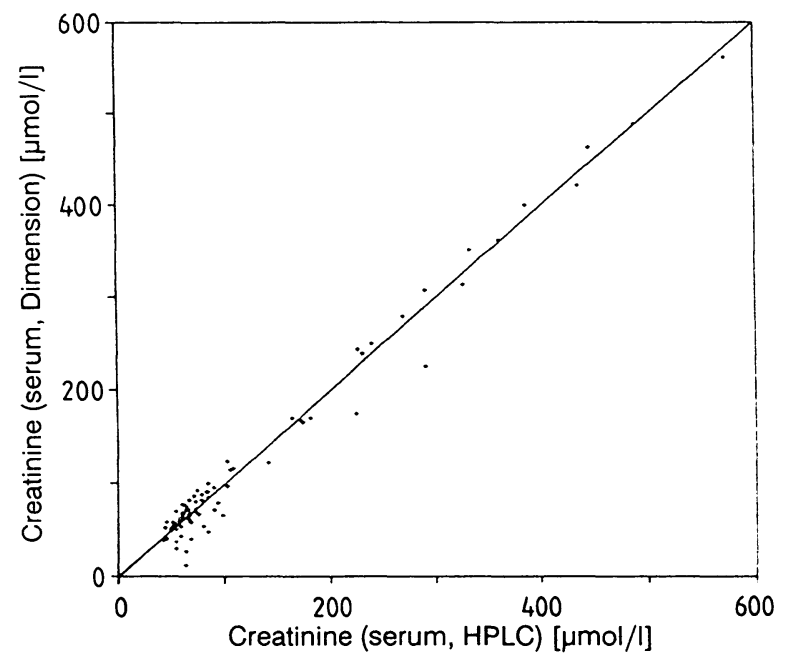

Fig. 3. Comparison of the Dimension creatinine method (yaxis) and a HPLC based reference method (x-axis) with 85 icteric samples (see Results).

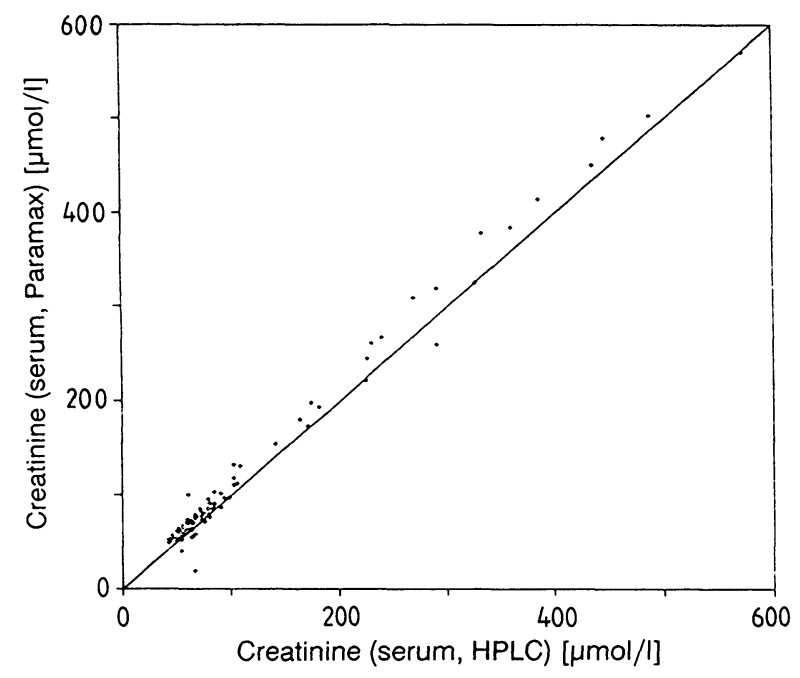

Fig. 4. Comparison of the Paramax creatinine method (y-axis) and a HPLC based reference method (x-axis) with 85 icteric samples (see Results).

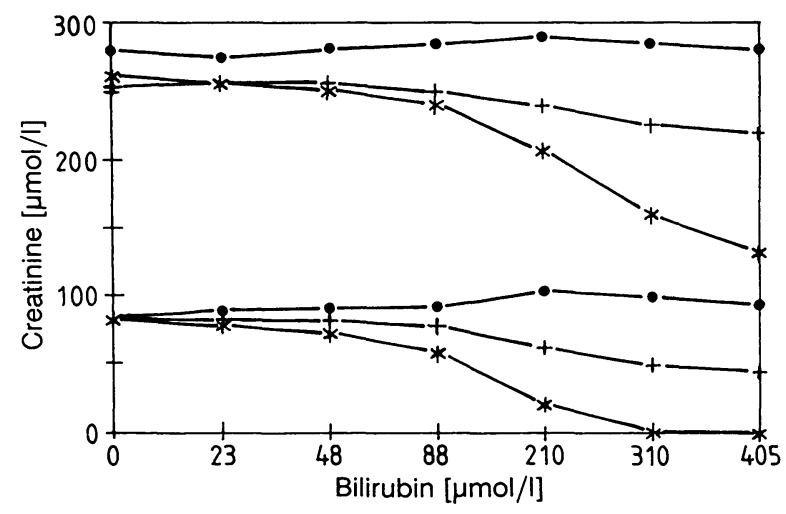

Fig. 5. The influence of unconjugated bilirubin (stocked human albumin solutions) on the determination of creatinine with three analysers:

- = Paramax

$+=$ Dimension

* $=$ Chem-1 (current)
Evaluation of the Chem-1 creatinine modifi cation

It is beyond the scope of this article to report extensively on the analytical aspects of the new method. Briefly, we found the following:

ninety-six, non-icteric specimens, covering an equally distributed range from $50-1500 \mu \mathrm{mol} / \mathrm{l}$ were measured with both cassettes (current and new) at the same time, and separately by HPLC. For calibration of the Chem-1 the same slope and intercept for both methods were used. Both methods were found to be linear over this range and the correlations with HPLC excellent and almost identical. The coefficients of variation, measured over a 10-day period, were 2.9 vs $2.5 \%$ (current vs new) for the lower value $(140 \mu \mathrm{mol} / \mathrm{l})$ and 1.9 vs $1.5 \%$ for the higher $(750 \mu \mathrm{mol} / \mathrm{l})$.

Table 2 gives information on the interference of bilirubin (unconjugated), haemoglobin, lipids, acetone and ascorbic acid.

Tab. 2. Some interference data on both Chem-1 formulations

\begin{tabular}{lll}
\hline & Chem-1 (current) & Chem-1 (new) \\
\hline Bilirubin & + & + \\
Haemoglobin & \pm & \pm \\
Lipids & $\left.+^{1}\right)$ & $\left.+^{1}\right)$ \\
Acetone & 0 & 0 \\
Ascorbic acid & + & \pm \\
\hline
\end{tabular}

1) Influence moderate at above normal creatinine concentrations

In addition, 144 icteric patient samples were studied with bilirubin concentrations ranging from 58 to 564 $\mu \mathrm{mol} / \mathrm{l}$. In figures 6 and 7 the comparisons of both cassettes with HPLC are shown. The bilirubin contents are given in table 1.

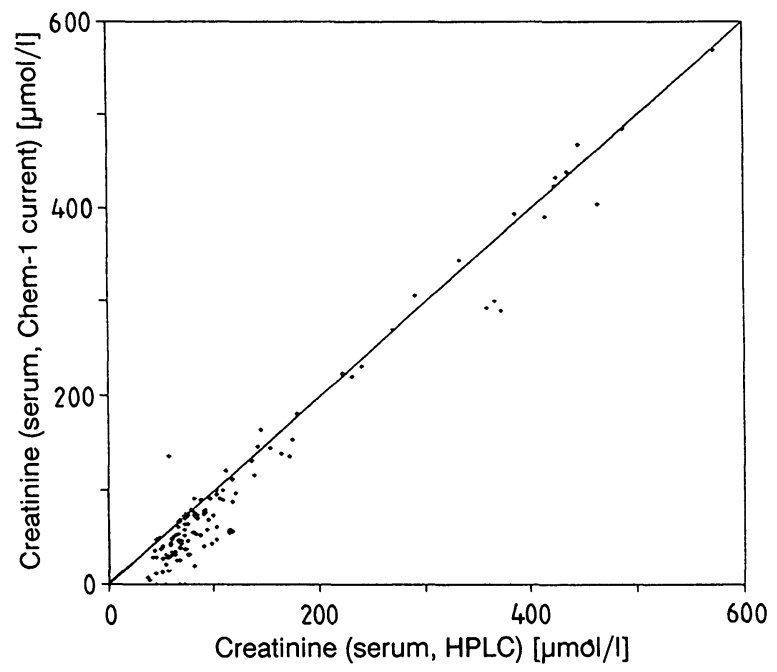

Fig. 6. Comparison of the current Chem-1 creatinine formulation ( $y$-axis) and a HPLC based reference method ( $x$ axis) with 144 icteric samples (see Results). 


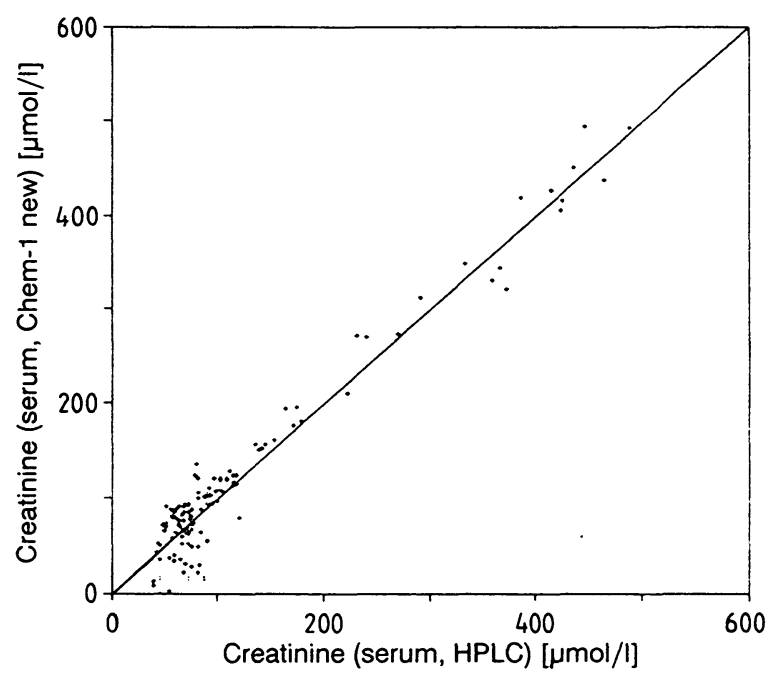

Fig. 7. Comparison of the new Chem-1 creatinine formulation (y-axis) and a HPLC based reference method ( $x$-axis) with 144 icteric samples (see Results).

Comparison two creatinine methods on the Dimension

Ninety-nine icteric samples (the total bilirubin contents are also given in tab. 1) were compared with HPLC, the results of which are depicted in figures 8 and 9.

\section{Discussion}

This study was initiated for practical reasons. We were faced by situations, in which the results of our routine creatinine determination did not fit the clinical picture. Sometimes the clinicians were right, sometimes they were not. In our laboratory we see serum samples with elevated bilirubin levels 5-10 times per day (total bilirubin higher than $50 \mu \mathrm{mol} / \mathrm{l}$ ). Normally only total bilirubin is requested. Therefore only these results were used and no effort was made at this point to subdivide into bilirubin fractions. This may be worthwhile in a future study.

It is known that the Jaffé reaction is susceptible to interference. The latest edition of Young's "Effects of Drugs on Clinical Laboratory Tests" contains a number of pages on this problem (7). From this list it is very clear that bilirubin, the starting-point of our study, is not the only interferent. This is also what we experienced, and it makes the problem even more frustrating. However, the visible colour of elevated bilirubin in serum is a good means of selecting samples for study.

It is known from the literature that the speed of analysis is improved by measuring the rate of the Jaffé reaction, rather than its end point (8). There are

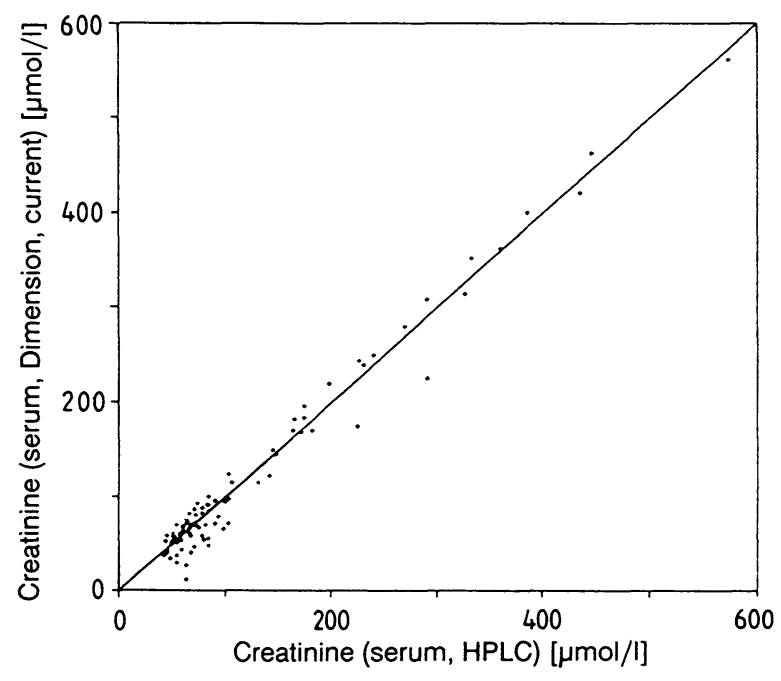

Fig. 8. Comparison of the current Dimension creatinine method (y-axis) and a HPLC based reference method (x-axis) with 99 icteric samples (see Results).

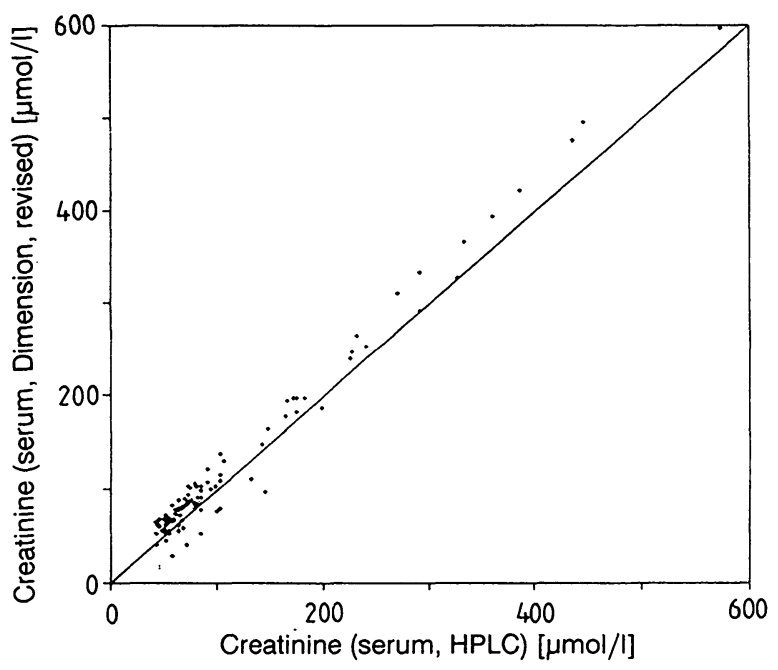

Fig. 9. Comparison of the revised Dimension creatinine method (y-axis) and a HPLC based reference method (x-axis) with 99 icteric samples (see Results).

also reports describing the circumvention of the influence of some interferents. Important factors mentioned are: picrate and base concentrations, reaction time and bichromatic measurement $(9-12)$. Modern, high-volume analysers employing the Jaffé reaction can only use a kinetic version of the analysis. The reaction conditions represent a compromise designed to fulfil certain criteria with regard to speed of analysis, accuracy and precision. Sometimes the user is left with a flagged result indicating that there is a problem. In cases of interference it generally means that a back-up method is needed. 
From figures 2, 3, 4, and 5 it is clear that the current Chem-1 creatinine method has the largest bias with our set of icteric specimens and that, with some exceptions, the Paramax results seem the most accurate, although the differences between the Paramax and the Dimension are generally not very pronounced. Although the numerical values are not given, it is striking that strongly deviating results in one instrument are often correct in the other two analysers. In our opinion this can be attributed to the reaction conditions, which differ considerably in all instruments.

Concerning the new Chem-1 creatinine formulation, it is quite clear from figures 6 and 7 that there is an improvement, although there appears to be some kind of overcompensation. It is still disappointing that, especially in the lower range, which is clinically important, a number of outliers still exists.

Technicon claims that the new formulation, which will be commercially available in 1992, also has improved stability. This may be true, but we have not thoroughly checked the stability of the formulation, because of the limited length of our study and also because we focussed our attention on the interference of bilirubin. Regarding the analytical performance for non-icteric specimens, both cassettes are more or less comparable in our opinion and also acceptable.

The question arises of whether we will be satisfied when the new formulation, together with the new software, is released. The answer is no. This can best be explained by considering table 3 and figure 10 .

Tab. 3. Results obtained from 10 neonatal serum pools. All results are expressed in $\mu \mathrm{mol} / 1$

\begin{tabular}{rllll}
\hline Part & HPLC & $\begin{array}{l}\text { Chem-1 } \\
\text { (current) }\end{array}$ & $\begin{array}{l}\text { Chem-1 } \\
\text { (new) }\end{array}$ & $\begin{array}{l}\text { Total } \\
\text { bilirubin }\end{array}$ \\
\hline 1 & 49 & 4 & 39 & 176 \\
2 & 50 & 17 & 29 & 134 \\
3 & 50 & 35 & 80 & 104 \\
4 & 55 & 44 & 80 & 114 \\
5 & 58 & 30 & 86 & 113 \\
6 & 55 & 34 & 76 & 109 \\
7 & 54 & 37 & 73 & 108 \\
8 & 48 & 31 & 66 & 118 \\
9 & 47 & 23 & 25 & 130 \\
10 & 43 & 7 & 16 & 160 \\
\hline
\end{tabular}

In table 3 the various data on ten neonatal specimens are given. It is clear that the results of both cassettes are totally unacceptable. Figure 10 gives an exploded view of figure 7 and shows a very critical range of creatinine values, clinically speaking.

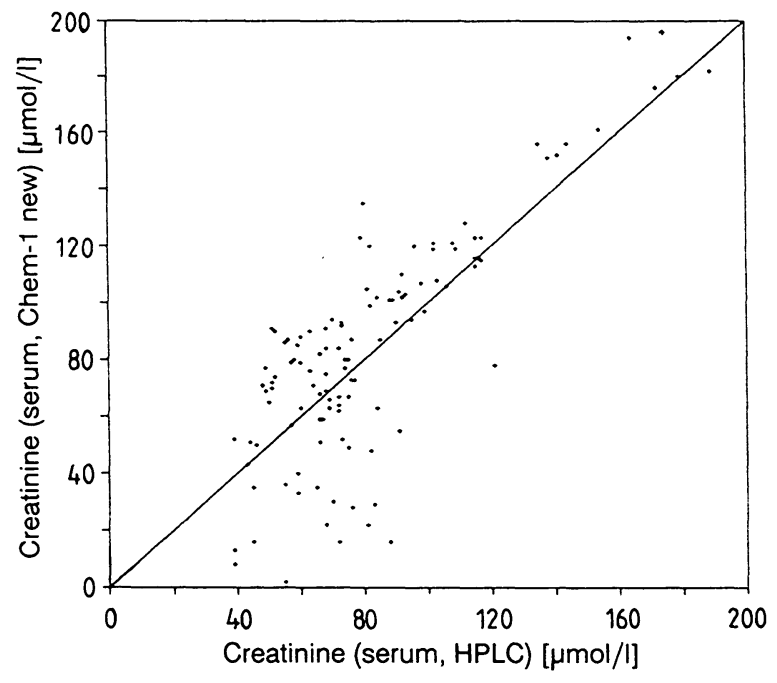

Fig. 10. Exploded part of figure 7.

Concerning the software the Chem-1 has a number of possibilities which can be related to the reaction conditions and which can be studied. We therefore discussed the results of our study with Technicon which resulted in the development of modified software in order to improve the bichromatic correction. With the same modified reagent formulation and this specially developed software we found in a pre-study performed with 82 icteric speciments (total bilirubin ranging from 104 up to $570 \mu \mathrm{mol} / \mathrm{l}$ ) a much better correlation with HPLC. These and the measurements regarding the influence of other interfering factors will be published later on.

The same holds true for the Dimension. Looking from a "high" standpoint figures 8 and 9 are comparable, i.e. many accurate results and a small number of outliers. More detailed information from the numerical values reveals that deviating results in one versions are correct in the other. So in fact a comparable invitation to DuPont.

The overall conclusion based on our findings described above is rather negative judging from professional standards on accuracy in relation with the analysis of all patient samples, no matter what the interfering factor may be. In this respect we can concur with Spencer who, in his excellent review article on the analytical aspects of the creatinine estimation, concludes by saying:

"Whilst kinetic methods in general have brought about marginal improvements in precision and specificity, it is probably fair to say that these methods still suffer from interference problems which are unlikely to be resolved by the kinetic approach" (8).

$\mathrm{He}$ expects that the solution will come by applying some kind of enzymatic approach. He may be right 
although his expectation has not been yet fulfilled at the moment (13). In the mean time, by filling the gap between the state of the art now and the ideal future solution, it is still worthwile to consider bichromatic correction mechanisms by applying various algorithms because there are no other ways at the moment (12). Modern analysers equipped with sophisticated computer facilities ought to be capable of these items. It should be stressed that quantitative knowledge on the analytical influence of at least the most common interferents, and hopefully the absence of this influence, in the modern closed systems, is needed.

\section{References}

1. Blijenberg, B. G., Boerma, G. J. M. \& Leijnse, B. (1989) An evaluation of the improved Technicon Chem-1 system. J. Clin. Chem. Clin. Biochem. 27, 457-469.

2. Egense, J., Koch, C. J. W. \& Willems, M. (1990) Adsorption of creatinine to Fuller's earth. Scand. J. Clin. Lab. Invest. $50,687-692$.

3. Zwang, L. \& Blijenberg, B. G. (1991) Assessment of a selected method for creatinine with special emphasis on bilirubin interference. Eur. J. Clin. Chem. Clin. Biochem. $29,795-800$.

4. Van Suijlen, J. D. E., Blijenberg, B. G., Boerma, G. J. M. \& Leijnse, B. (1991) Calibration of Technicon Chem-1 multitest analysers. J. Clin. Chem. Clin. Biochem. 29, 205208.

5. Levy, S., Rutman, M. \& Shikabi, Z. (1990) A novel bilirubin interference correction technique applied to the Technicon Chem-1. Clin. Chem. 36, 1113-1114.

6. Société Française de Biologie Clinique. Commission "Validation de Techniques - Protocol de validation de techniques (1986) Ann. Biol. Clin. 44, 686-745.

7. Young, D. S. (1990) Effects of drugs on clinical laboratory tests, AACC Press, Washington D.C. 20006.

\section{Acknowledgements}

Thanks are due to Bayer-Technicon, especially the Tarrytown branch, for supplying the reagents and the software of the new method. In this respect the stimulating discussions with Dr. $J$. Levine (Bayer-Technicon, U.S. A.) need to be mentioned.

The revision of the Dimension software was done by Mr. $G$. Salemink (DuPont, The Netherlands).

For the Paramax measurements we want to thank Dr. J. M. Pekelharing (Stichting Samenwerkende Ziekenhuizen, Delft).

Analytical support was given by $R$. J. Leeneman, $C$. J. M. van Leeuwen and $L$. J. Perret and clerical assistance by $A$. CopperStaamer.

8. Spencer, K. (1986) Analytical reviews in clinical biochemistry: the estimation of creatinine. Ann. Clin. Biochem. 23, $1-25$.

9. Bowers, L. D. (1980) Kinetic serum creatinine assays I. The role of various factors in determining specificity. Clin. Chem. 26, $551-554$.

10. Bowers, L. D. \& Wong, E. T. (1980) Kinetic serum creatinine assays II. A critical evaluation and review. Clin. Chem. $26,555-561$

11. Pardue, H. L., Bacon, B. L., Groeger Nevius, M. \& Skoug, J. W. (1987) Kinetic study of the Jaffé reaction for quantifying creatinine in serum: 1 . Alkalinity controlled with $\mathrm{NaOH}$. Clin. Chem. 33, 278-285.

12. Bacon, B. L. \& Pardue, H. L. (1989) Kinetic study of the Jaffé reaction for quantifying creatinine in serum: 2. Evaluation of buffered reagent and comparison of different data-processing options. Clin. Chem. 35, 360-363.

13. Weber, J. A. \& Van Zanten, A. P. (1991) Interferences in current methods for measurements of creatinine. Clin. Chem. 37, 695-700.

Dr. B. G. Blijenberg

Academic Hospital Rotterdam-Dijkzigt

Department of Clinical Chemistry

Dr. Molewaterplein 40

NL-3015 GD Rotterdam

The Netherlands 